\title{
Orthogonality of generalized eigenfunctions in Weyl's expansion theorem
}

\author{
By
}

\author{
Michihiko Matsuda
}

\section{§. Introduction}

Let us consider an ordinary differential equation of the second order

$$
\frac{d^{2} u}{d x^{2}}-q(x) u(x)+\lambda u(x)=0, \quad(0 \leqq x<\infty)
$$

Here $q(x)$ is a real-valued function which is locally summable and $x=0$ is a regular point of the equation. Let $\varphi(x: \lambda)$ be the solution of the equation which satisfies

$$
\varphi(0 ; \lambda)=\sin \theta, \frac{\partial \varphi}{\partial x}(0 ; \lambda)=\cos \theta
$$

where $\theta$ is a real constant.

H. Weyl proved that there exists a spectral measure $\rho_{0}(\lambda)$ which satisfies the following two conditions (A) and (B) (Weyl [14]):

(A) The transformation

$$
\mathscr{F}_{\rho_{0}}: f(x) \rightarrow \int_{0}^{\infty} f(x) \varphi(x ; \lambda) d x
$$

from $L_{2}(0, \infty ; d x)$ into $L_{2}\left(-\infty,+\infty ; d \rho_{0}\right)$ is isometric, namely

$$
\int_{-\infty}^{+\infty}\left|\mathscr{F}_{\rho_{0}} f(\lambda)\right|^{2} d \rho_{0}(\lambda)=\int_{0}^{\infty}|f(x)|^{2} d x
$$

(B) $\mathscr{F}_{\rho_{0}}$ transforms $L_{2}(0, \infty ; d x)$ onto $L_{2}\left(-\infty,+\infty ; d \rho_{0}\right)$.

Such a spectral measure is unique ${ }^{(1)}$ and is called the Weyl spectral measure.

Let us call a spectral measure $\rho(\lambda)$ which only satisfies (A) a pseudospectral measure.

Received June 1, 1966 
I. M. Gelfand and B. M. Levitan succeeded in determining the potential function $q(x)$ from a given spectral measure $\rho(\lambda)$. In general, however, $\rho(\lambda)$ turns out to be a pseudo-spectral measure for the equation with the $q(x)$ thus determined. They gave a sufficient condition for $\rho(\lambda)$ to be the Weyl spectral measure. In particular, if $\rho(\lambda)$ vanishes for large $-\lambda, \rho(\lambda)$ will be the Weyl spectral measure (Gelfand-Levitan [4]).

Also M. G. Krein gave a sufficientt and necessary condition for a pseudo-spectral measure to be the Weyl spectral measure (Krein [7]). Previous to them V. A. Marčenko proved that $q(x)$ is uniquely determined by $\rho(\lambda)$ (Marčenko [9]).

We are interested in the condition which $q(x)$ should satisfy in order that every pseudo-spectral measure of the equation be the Weyl spectral measure.

We shall prove in $\S 1$ that, in the limit-point case at infinity, a pseudospectral measure is the Weyl spectral measure and that, in the limit-circle case at infinity, there always exists a pseudo-spectral measure which is not the Weyl spectral measure.

Combining this theorem with the result of Gelfand-Levitan, we see that the spectrum in the limit-circle case at infinity is always unbounded below $(\S 2)$.

K. O. Friedrichs stated that the following theorem was a result of Rellich (Friedrichs [3]):

Let $q(x)$ be continuous and negative on $[0, \infty]$. Then, if $q(x) \rightarrow-\infty$ as $x \rightarrow \infty$ and if

$$
\int^{\infty}|q(x)|^{-1 / 2} d x<\infty,
$$

the spectrum is discrete and unbounded below.

Also D. B. Sears and E. C. Titchmarsh obtained the following result: if the inequality $(0.3)$ holds, the equation is of the limit-circle type at infinity and hence the spectrum is discrete (Sears-Titchmarsh [11], Titchmarsh [13]). Our theorem gave a proof of lower unboundedness of the spectrum. Proposition 3 in $\S 2$ is due to Y. Saitō.

(1) In the limit-circle case at infinity we set a boundary condition at infinity. 
The author wishes to express his gratitude to Professors K. Ito, H. Yoshizawa and T. Ikebe for their continuous interest and encouragement in the course of his writing this note. He also feels grateful to his friend Y. Saitō for fruitful discussions with him.

\section{$\S 1$. Properties of the pseudo-spectral measure}

Let $\mathscr{D}_{0}$ be the class of all functions $f(x)$ which satisfy the following four conditions (i). . (iv):

(i) $f(x)$ has a compact carrier.

(ii) $f(x)$ is expressed in the form

$$
f(x)=\int_{0}^{x} d y \int_{0}^{y} g(z) d z+c
$$

where $g(z)$ is a locally summable function and $c$ is a constant.

(iii) $-g(x)+q(x) f(x)$ belongs to $L_{2}(0, \infty ; d x)$.

(iv) $f(0) \cos \theta-f^{\prime}(0) \sin \theta=0$.

We shall define a symmetric operator $L_{0}$ with the domain $\mathscr{D}_{0}$ by

$$
L_{0 .} f(x)=-g(x)+q(x) f(x) .
$$

Let us define a pseudo-spectral measure as a spectral measure $\rho(\lambda)$ which satisfies the following identity for every $f(x)$ in the class $\mathcal{C}_{0}$ of all continuous functions each of which has a compact carrier,

$$
\int_{0}^{\infty}|f(x)|^{2} d x=\int_{+\infty}^{+\infty}\left|\int_{0}^{\infty} f(x) \varphi(x ; \lambda) d x\right|^{2} d \rho(\lambda) .
$$

Let us denote $\int_{0}^{\infty} f(x) \varphi(x ; \lambda) d x$ by $\mathscr{F}_{0} f(\lambda)$ for $f(x)$ in $\mathcal{C}_{0}$. Then we have an isometric transformation $\mathscr{I}_{0}$ from $\mathcal{C}_{0}$ into $L_{2}(-\infty,+\infty ; d \rho)$. Since $\mathcal{C}_{0}$ is dense in $L_{2}(0, \infty ; d x)$, we can extend $\mathscr{F}_{0}$ to the isometric transformation $\mathscr{F}_{\rho}$ which is defined on $L_{2}(0, \infty ; d x)$. $\mathscr{F}_{\rho}$ is expressed for every function $h(x)$ in $L_{2}(0, \infty ; d x)$ in the form

$$
\mathscr{L}_{\mathrm{p}} h(\lambda)=1 . \underset{N \rightarrow \infty}{\mathrm{i} . \mathrm{m}} \cdot \int_{0}^{N} h(x) \varphi(x ; \lambda) d x
$$

We shall define a bounded linear transformation $\mathscr{L}_{\rho}^{*}$ from $L_{2}(-\infty$, 
$+\infty ; d \rho)$ onto $L_{2}(0, \infty ; d x)$ by

$$
\left\langle\mathscr{F}_{p}^{*} \xi, f\right\rangle=\left\langle\xi, \mathscr{F}_{p} f\right\rangle_{p} .
$$

Here $\langle$,$\rangle and \langle,\rangle_{\rho}$ are the inner products in $L_{2}(0, \infty ; d x)$ and $L_{2}(-\infty$, $+\infty ; d \rho)$, respectively.

We have $\left\|\mathscr{F}_{p}^{*} \xi\right\| \leqq\|\xi\|_{\rho}$.

Putting $\xi=\mathscr{F}_{p} h$ in (1.4), we have

$$
\left\langle\mathscr{F}_{p}^{*} \mathscr{F}_{\rho} h, f\right\rangle=\left\langle\mathscr{F}_{\rho} h, \mathscr{F}_{\rho} f\right\rangle_{\rho}=\langle h, f\rangle .
$$

Hence it follows that $\mathscr{F}_{\rho}^{*} \mathscr{F}_{\rho}$ is the identity operator.

Proposition 1. Let $\rho(\lambda)$ be a pseudo-spectral measure. Then $\mathscr{F}_{p}^{*}$ is expressed in the form

$$
\mathscr{F}_{P}^{*} \xi(x)=\int_{-\infty}^{+\infty} \xi(\lambda) \varphi(x, \lambda) d \rho(\lambda)
$$

for every continuous function $\xi(\lambda)$ which has a compact carrier.

Proof. By the definition we have

$$
\int_{0}^{\infty} \mathscr{F}_{p}^{*} \xi(x) \overline{f(x)} d x=\int_{-\infty}^{+\infty} \xi(\lambda) \overline{\mathscr{F}_{\rho} f(\lambda)} d \rho(\lambda) .
$$

Suppose that $f(x)$ belongs to $\mathcal{C}_{0}$. Then we get

$$
\begin{aligned}
& \int_{-\infty}^{+\infty} \xi(\lambda) \overline{\mathscr{F}_{\rho}} f(\bar{\lambda}) d \rho(\lambda)=\int_{-\infty}^{+\infty} \xi(\lambda) d \rho(\lambda) \int_{0}^{\infty} \overline{f(x)} \varphi(x ; \lambda) d x \\
& =\int_{0}^{\infty} \overline{f(x)} d x \int_{-\infty}^{+\infty} \xi(\lambda) \varphi(x ; \lambda) d \rho(\lambda) .
\end{aligned}
$$

Hence we obtain

$$
\int_{0}^{\infty} \overline{f(x)} d x\left\{\mathscr{F}_{\rho}^{*} \xi(\lambda)-\int_{-\infty}^{+\infty} \xi(\lambda) \varphi(x ; \lambda) d \rho(\lambda)\right\}=0 .
$$

Since this identity holds for every $f(x)$ in $\mathcal{C}_{0}$, we have the proposition.

Corollary. Let $\rho(\lambda)$ be a pseudo-spectral measure. Then for every $h(x)$ in $L_{2}(0, \infty ; d x)$ we have

$$
h(x)=1 \text {. i. } \operatorname{m} . \int_{-N}^{N} \mathscr{F}_{\rho} h(\lambda) \varphi(x ; \lambda) d \rho(\lambda) .
$$


Orthogonality of generalized eigenfunctions in Weyl's expansion 247

The following three conditions are equivalent:

(i ) $\mathscr{F}_{p} \mathscr{F}_{p}^{*}=$ identity.

(ii) $\mathscr{F}_{P}^{*}$ is injective.

(iii) $\mathscr{F}_{p}$ is surjective.

Theorem 1. In the limit-circle case at infinity there always exists a pseudo-spectral measure which is not the Weyl spectral measure. In the limit-point case at infinity any pseudo-spectral measure $\rho(\lambda)$ is the Weyl spectral measure.

Proof. In the limit-circle case at infinity we can set two different boundary conditions at infinity. According to these boundary conditions we have two different Plancherel's identities

$$
\begin{aligned}
& \|h\|^{2}=\sum_{n=1}^{\infty}\left|\left\langle h, \varphi\left(\cdot ; \lambda_{n}\right)\right\rangle\right|^{2} c_{n}, \\
& \|h\|^{2}=\sum_{m=1}^{\infty}\left|\left\langle h, \varphi\left(\cdot ; \lambda_{m}^{\prime}\right)\right\rangle\right|^{2} c_{m}^{\prime} .
\end{aligned}
$$

Hence we have

$$
\begin{aligned}
\|h\|^{2}= & \sum_{n=1}^{\infty}\left|\left\langle h, \varphi\left(\cdot ; \lambda_{n}\right)\right\rangle\right|^{2} \frac{c_{n}}{2} \\
& +\sum_{m=1}^{\infty}\left|\left\langle h, \varphi\left(\cdot ; \lambda_{m}^{\prime}\right)\right\rangle\right|^{2} \frac{c_{m}^{\prime}}{2} .
\end{aligned}
$$

Let $\rho(\lambda)$ be the measure defined by

$$
\int_{\Delta} d \rho(\lambda)=\sum_{\lambda_{n} \in \Delta} \frac{c_{n}}{2}+\sum_{\lambda^{\prime} m \in \Delta} \frac{c_{m}^{\prime}}{2} .
$$

Then $\rho(\lambda)$ is a pseudo-spectral measure.

However, no function $h(x)$ in $L_{2}(0, \infty ; d x)$ can satisfy $\left\langle h, \varphi\left(\cdot ; \lambda_{1}\right)\right\rangle$ $=1$ and $\left\langle h, \varphi\left(\cdot ; \lambda_{m}^{\prime}\right)\right\rangle=0$ (for every $\left.m\right)$ simultaneously, because $\left\{\varphi\left(x ; \lambda_{m}^{\prime}\right)\right\}_{m=1}^{\infty}$ is a complete system. Hence $\rho(\lambda)$ is not the Weyl spectral measure.

In the limit-point case at infinity the operator $L_{0}$ is essentially selfadjoint. The class $\mathcal{R}_{\mu}$ of all functions which can be expressed in the form $h(x)=\left(\mu-L_{0}\right) f(x)$ with $f(x)$ in $\mathscr{D}_{0}$ is dense in $L_{2}(0, \infty ; d x)$ for every complex number $\mu$ that is not real. 
Let $\rho(\lambda)$ be a pseudo-spectral measure. Then for $h(x)$ in $\mathcal{R}_{\mu}$ we get

$$
\mathscr{F}_{p} h(\lambda)=\mathscr{F}_{\rho}\left(\mu-L_{0}\right) f(\lambda)=(\mu-\lambda) \mathscr{F}_{\rho} f(\lambda) .
$$

Put $\boldsymbol{L}_{\mu}=\left(\mu-\bar{L}_{0}\right)^{-1}$. Then, by the identity $\boldsymbol{L}_{\mu} h(x)=f(x)$ we have

$$
\left\langle\boldsymbol{L}_{\mu} h, h\right\rangle=\int_{-\infty}^{+\infty} \frac{\left|\mathscr{F}_{p} h(\lambda)\right|^{2}}{\mu-\lambda} d \rho(\lambda) .
$$

This identity holds for every $h(x)$ in $L_{2}(0, \infty ; d x)$, because $\mathcal{R}_{\mu}$ is dense in $L_{2}(0, \infty ; d x)$.

For every finite interval $\Delta=(a, b)$, let us define the bounded symmetric operator $E_{\Delta}$ by

$$
\left\langle E_{\Delta} h, h\right\rangle=\lim _{\varepsilon_{\downarrow} 0} \frac{1}{2 \pi i} \int_{c_{\varepsilon}}\left\langle\boldsymbol{L}_{\mu} h, h\right\rangle d \mu,
$$

where $c_{\varepsilon}$ is the following path of integration.

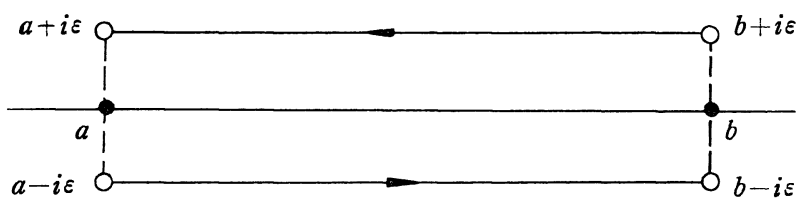

Then $E_{\Delta}$ proves to be a projection in $L_{2}(0, \infty ; d x){ }^{(1)}$

Let $P_{\Delta}$ denote the projection defined by

$$
P_{\Delta} \xi(\lambda)=\left\{\begin{array}{cc}
\xi(\lambda), & \lambda \in \Delta \\
0, & \lambda \notin \Delta .
\end{array}\right.
$$

First we shall prove the identity

$$
\mathscr{F}_{\rho} E_{\Delta}=P_{\Delta} \mathscr{F}_{\rho}
$$

For every $f(x)$ in $\mathcal{C}_{0}$ we have by (1.7) and (1.8)

$$
\left\langle E_{\Delta} f, f\right\rangle=\int_{\Delta}\left|\mathscr{F}_{\rho} f(\lambda)\right|^{2} d \rho(\lambda) .
$$

Writing this as

$$
\left\langle E_{\Delta} f, f\right\rangle=\left\langle P_{\Delta} \mathscr{F}_{\rho} f, \mathscr{F}_{\rho} f\right\rangle_{\rho},
$$

we get

$$
\left\langle E_{\Delta} f_{1}, f_{2}\right\rangle=\left\langle P_{\Delta} \mathscr{F}_{\rho} f_{1}, \mathscr{F}_{\rho} f_{2}\right\rangle_{\rho}
$$

(1) Stone [12], Theorem 5.6. 
Orthogonality of generalized eigenfunctions in Weyl's expansion 249 for every pair of $f_{1}(x)$ and $f_{2}(x)$ in $\mathcal{C}_{0}$.

For $f(x)$ in $\mathcal{C}_{0}$, put $\xi(\lambda)=\mathscr{E}_{p} E_{\Delta} f(\lambda)-P_{\Delta} \mathscr{F}_{p} f(\lambda)$. Then we get by (1.11)

$$
\begin{aligned}
& \left\langle\xi, \mathscr{F}_{\rho} f_{1}\right\rangle_{\rho}=\left\langle\mathscr{F}_{\rho} E_{\Delta} f-P_{\Delta} \mathscr{F}_{\rho} f, \mathscr{F}_{\rho} f_{1}\right\rangle_{\rho} \\
& =\left\langle E_{\Delta} f, f_{1}\right\rangle-\left\langle P_{\Delta} \mathscr{F}_{\rho} f, \mathscr{F}_{\rho} f_{1}\right\rangle_{\rho}=0 .
\end{aligned}
$$

Hence the identity

$$
\left\|P_{\Delta} \mathscr{F}_{\rho} f\right\|_{\rho}^{2}=\left\|\mathscr{F}_{\rho} E_{\Delta} f\right\|_{\rho}^{2}+\|\xi\|_{\rho}^{2}
$$

holds, because $\xi(\lambda)$ and $\mathscr{F}_{\rho} E_{\Delta} f(\lambda)$ are othhogonal. Since $E_{\Delta}$ is a projection, we have

$$
\left\langle E_{\Delta} f, f\right\rangle=\left\|E_{\Delta} f\right\|^{2}=\left\|\mathscr{F}_{\rho} E_{\Delta} f\right\|_{\rho}^{2} .
$$

On the other hand, since $P_{\Delta}$ is a projection, we have by (1.10)

$$
\left\langle E_{\Delta} f, f\right\rangle=\left\langle P_{\Delta} \mathscr{F}_{\rho} f, \mathscr{F}_{\rho} f\right\rangle_{\rho}=\left\|P_{\Delta} \mathscr{F}_{\rho} f\right\|_{\rho}^{2} .
$$

Comparing (1.13) with (1.14), we get

$$
\left\|\mathscr{F}_{\rho} E_{\Delta} f\right\|_{\rho}^{2}=\left\|P_{\Delta} \mathscr{F}_{\rho} f\right\|_{\rho}^{2} .
$$

Hence we obtain from (1.12) the identity

$$
\|\xi\|_{\rho}^{2}=0 \text {. }
$$

Since $\mathcal{C}_{0}$ is dense in $L_{2}(0, \infty ; d x)$, the identity (1.9) is proved.

Suppose that a function $\eta(\lambda)$ in $L_{2}(-\infty,+\infty ; d \rho)$ satisfies $\left\langle\eta, \mathscr{F}_{\rho} f\right\rangle_{\rho}=0$ for every $f(x)$ in $L_{2}(0, \infty ; d x)$. We prove that $\eta=0$ in $L_{2}(-\infty,+\infty ; d \rho)$. It follows from (1.9) that $P_{\Delta} \mathscr{F}_{p} f(\lambda)$ belongs to the image of $\mathscr{L}_{p}$ for every $f(x)$ in $\mathcal{C}_{0}$. Hence for every finite interval $\Delta$ we have

$$
\left\langle\eta, P_{\Delta} \mathscr{F}_{\rho} f\right\rangle_{\rho}=\int_{\Delta} \eta(\lambda) \overline{\mathscr{F}_{\rho} f(\lambda)} d \rho(\lambda)=0 .
$$

Since the inequality

$$
\int_{-\infty}^{+\infty}\left|\eta(\lambda) \mathscr{F}_{\rho} f(\lambda)\right| d \rho(\lambda)<\infty
$$

holds, we obtain

$$
\int_{-\infty}^{+\infty}\left|\eta(\lambda) \mathscr{F}_{\rho} f(\lambda)\right| d \rho(\lambda)=0
$$

For every $\lambda_{0}$ in $(-\infty,+\infty)$ we can take such a function $f_{0}(x)$ in $\mathcal{C}_{0}$ that 
$\mathscr{I}_{p} f_{0}\left(\lambda_{0}\right) \neq 0$. Since $\mathscr{I}_{p} f_{0}(\lambda)$ is a continuous function of $\lambda, \eta(\lambda)$ vanishes on a neighbourhood of $\lambda_{0}$. Hence we have $\eta=0$ in $L_{2}(-\infty,+\infty ; d \rho)$.

Remark 1. K. Yosida constructed a pseudo-spectral measure with the classical method of Hilbert (Yosida [15], Hilbert [5]).

Remark 2. The second part of our theorem can be proved by the method E. A. Coddington and N. Levinson used to prove Weyl's theorem in the limit-point case at infinity. (Coddington-Levinson [1], 239-242). See also Ikebe [6].

Remark 3. If we assume the existence of the Weyl spectral measure, we can prove the second part of our theorem more easily using Lemma 2 in Saitō [10] $§ 1$. See Remark in Saitō [10] $\S 1$.

\section{§2. Pseudo-spectral measures in the limit-circle case at infinity}

The spectrum in the limit-circle case at infinity is discrete (Weyl [14]). Now we shall prove

Theorem 2. The spectrum in the limit-circle case at infinity is always unbounded below.

Proof. We parametrize boundary conditions at infinity with $\alpha$ which varies over $[0,2 \pi)$. Let $\rho_{x}(\lambda)$ be the Weyl spectral measure under the boundary condition parametrized with $\alpha$.

First we shall prove that there exists at most one value of $\alpha$ for which $\rho_{\alpha}(\lambda)$ vanishes for large $-\lambda$. Suppose in contrary that there exist two such values $\alpha_{1}$ and $\alpha_{2}$. Then we could construct from $\rho_{\alpha_{1}}(\lambda)$ and $\rho_{a_{2}}(\lambda)$ a pseudo-spectral measure $\rho(\lambda)$ which is not the Weyl spectral measure and vanishes for large $-\lambda$. But this is impossible, because by a result of Gelfand-Levitan (Gelfand-Levitan [4], Levitan [8]) every pseudo-spectral measure that vanishes for large $-\lambda$ is the Weyl spectral measure.

If there exists a parameter $\alpha_{0}$ such that $\rho_{\rho_{0} 0}(\lambda)$ vanishes for large $-\lambda$, we have for every $f(x)$ in $\mathscr{D}_{0}$

$$
\left\langle L_{0} f, f\right\rangle \geqq c\|f\|^{2},
$$


where $c$ is a real constant. Then every self-adjoint extension of $L_{0}$ becomes lower bounded. ${ }^{(1)}$ Hence every $\rho_{a}(\lambda)$ vanishes for large $-\lambda$. This is a contradiction.

Corollary. For every pseudo-spectral measure $\rho(\lambda)$ in the limit-circle case at infinity, the support of the measure $d \rho$ is unbounded below.

Remark 1. E. C. Titchmarsh and D. B. Sears proved the following theorem:

$$
\text { Let (a) } \begin{aligned}
& q(x) \leqq 0, \quad q^{\prime}(x)<0, \\
& q(x) \rightarrow-\infty \text { as } x \rightarrow \infty, \\
& q^{\prime}(x)=O\left\{|q(x)|^{c}\right\}, \quad\left(0<c<\frac{3}{2}\right)
\end{aligned}
$$

and let (b) $q^{\prime \prime}(x)$ be ultimately of one sign. Then, if

$$
\int^{\infty}|q(x)|^{-1 / 2} d x<\infty
$$

the equation is of the limit-circle type at infinity and hence the spectrum is discrete (Titchmarsh [13], Theorem 5.11., Dunford-Schwartz [2], 1448., Sears-Titchmarsh [11]).

Combining our theorem with Sears-Titchmarsh's we have the following result:

Under the conditions (a) and (b), if the inequality (2.1) holds, then the spectrum is discrete and unbounded below. ${ }^{(2)}$

Remark 2. By Weyl's classification theorem, Theorem 2 is equivalent to the following: if the $L_{0}$ in $\S 1$ is bounded below, then the equation is of the limit-point type at infinity. In higher-dimensional cases, it is known that, if the operator $L_{0} \equiv-\Delta+V(x)$ is lower bounded, then it is essentially self-adjoint. However, in this case it is assumed that $V(x)$ is locally square summable, while in our case $q(x)$ is assumed to be locally summable. See Saitō [10] $§ 2$.

(1) Dunford-Schwartz [2], 1454.

(2) To prove this fact only, it is unnecessary to use Theorem 2 . The lower unboundedness of the spectrum in the case where $q(x) \rightarrow-\infty$ as $x \rightarrow \infty$, can be proved by an elementary method. This remark was given by K. Asano, 
The following proposition explains why a spectral measure which only satisfies (A) in $\S 0$ is called here a pseudo-spectral measure.

Let $\mathscr{R}(\rho)$ be the range of $\mathscr{F}_{\rho}$. Then $\mathscr{R}(\rho)$ is the Hilbert space. If both $\xi(\lambda)$ and $\lambda \xi(\lambda)$ belong to $\mathscr{R}(\rho)$, we set $\Lambda_{\rho} \xi(\lambda)=\lambda \xi(\lambda)$. The image of $\mathscr{D}_{0}$ by $\mathscr{F}_{\rho}$ is contained in the domain of $\Lambda_{\rho}$, because for every $f(x)$ in $\mathscr{D}_{0}$ we have

$$
\lambda \mathscr{F}_{p} f(\lambda)=\mathscr{F}_{p} L_{0} f(\lambda) \text {. }
$$

Hence $\Lambda_{\rho}$ is a closed symmetric operator in $\mathscr{R}(\rho)$.

Proposition 2. Let $\rho(\lambda)$ be a pseudo-spectral measure which is not the Weyl spectral measure. Then we have

$$
\mathscr{F}_{\rho}^{-1} \Lambda_{\rho} \mathscr{F}_{\rho}=\bar{L}_{0} \text {. }
$$

Here $\mathscr{F}_{\rho}^{-1}$ is the restriction of $\mathscr{F}_{\rho}^{*}$ on $\mathscr{R}(\rho)$.

Proof. We prove the following: if $L \equiv \mathscr{F}_{\rho}^{-1} \Lambda_{\rho} \mathscr{F}_{\rho}$ is self-adjoint, then $\rho(\lambda)$ is the Weyl spectral measure.

By virtue of a theorem of Stone (Stone [12], Theorem 10.17.) there corresponds a boundary condition at infinity to the self-adjoint $L$, because $L$ is an extension of $L_{0}$. Hence we have a complete orthogonal system of eignefunctions of $L,\left\{\varphi\left(x ; \lambda_{n}\right)\right\}_{n=1}^{\infty}$. By the definition we get

$$
\lambda_{n} \mathscr{F}_{p} \varphi_{n}(\lambda)=\lambda \mathscr{F}_{p} \varphi_{n}(\lambda),
$$

where $\varphi_{n}(x)=\varphi\left(x ; \lambda_{n}\right)$.

Since $\mathscr{F}_{\rho} \varphi_{n}(\lambda)=\left\langle\varphi_{n}, \varphi(\cdot ; \lambda)\right\rangle$ vanishes only on $\lambda_{m}(m \neq n)$, we have $\int_{I} d \rho=0$ for every $I$ that does not contain an eigenvalue of $L$.

$$
\begin{aligned}
& \text { Writing } \int_{\left\{\lambda_{m}\right\}} d \rho \text { as } c_{m} \text {, we have } c_{n}=\left\|\varphi_{n}\right\|^{-2} \text {, because } \\
& \qquad \begin{aligned}
\left\|\varphi_{n}\right\|^{2} & =\int_{-\infty}^{+\infty}\left|\mathscr{F}_{\rho} \varphi_{n}(\lambda)\right|^{2} d \rho(\lambda) \\
& =\sum_{m=1}^{\infty}\left|\mathscr{F}_{\rho} \varphi_{n}\left(\lambda_{m}\right)\right|^{2} c_{m}=\left|\mathscr{F}_{\rho} \varphi_{n}\left(\lambda_{n}\right)\right|^{2} c_{n} \\
& =\left\|\varphi_{n}\right\|^{4} c_{n} .
\end{aligned}
\end{aligned}
$$

Hence $\mathscr{F}_{\rho}$ is surjective and $\mathscr{R}(\rho)=L_{2}(-\infty,+\infty ; d \rho)$.

Remark. V. A. Marčenko proved that for every increasing function 
Orthogonality of generalized eigenfunctions in Weyl's expansion 253

$\rho(\lambda)$ there exists at most one equation of type (0.1) which has $\rho(\lambda)$ as a pseudo-spectral measure (Marčenko [9]). Hence if $\rho(\lambda)$ is a pseudospectral measure which is not the Weyl spectral measure of an equation of type (0.1), then $\rho(\lambda)$ can not be the Weyl spectral measure of any equation of type (0.1).

Let $\sigma(\alpha)$ be a measure on $[0,2 \pi)$ with $\int_{0}^{2 \pi} d \sigma=1$. Then we have the following identity for $f(x)$ in $\mathcal{C}_{0}$

$$
\|f\|^{2}=\int_{0}^{2 \pi} d \sigma(\alpha) \int_{-\infty}^{+\infty}\left|\mathscr{F}_{0} f(\lambda)\right|^{2} d \rho_{a}(\lambda) .
$$

Hence we obtain a pseudo-spectral measure $\pi_{\sigma}(\lambda)$ defined by

$$
\int_{\Delta} d \pi_{\sigma}(\lambda)=\int_{0}^{2 \pi} d \sigma(\alpha) \int_{\Delta} d \rho_{\alpha}(\lambda) .
$$

As to the inverse of this statement we only have the following

Proposition 3. ${ }^{(1)}$ Let $\left\{\varphi_{m}\right\}_{m=1}^{\infty}$ and $\left\{\psi_{n}\right\}_{n=1}^{\infty}$ be the complete orthonormal systems of eigenfunctions which correspond to different boundary conditions at infinity. If we have the identity for every $h(x)$ in $L_{2}(0, \infty ; d x)$

$$
\|h\|^{2}=\sum_{m=1}^{\infty}\left|\left\langle h, \varphi_{m}\right\rangle\right|^{2} a_{m}+\sum_{n=1}^{\infty}\left|\left\langle h, \psi_{n}\right\rangle\right|^{2} b_{n},
$$

where $a_{m}$ and $b_{n}$ are non-negative numbers, then we have $a_{m}=a, b_{n}=1-a$, for all $m$ and $n$.

Proof. By (2.4) we have

$$
\begin{aligned}
\langle f, h\rangle= & \sum_{m=1}^{\infty}\left\langle f, \varphi_{m}\right\rangle\left\langle\varphi_{m}, h\right\rangle a_{m} \\
& +\sum_{n=1}^{\infty}\left\langle f, \psi_{n}\right\rangle\left\langle\psi_{n}, h\right\rangle b_{n}
\end{aligned}
$$

for every pair of $f(x)$ and $h(x)$ in $L_{2}(0, \infty ; d x)$. Put $f(x)=\varphi_{k}(x)$ and $h(x)=\psi_{l}(x)$ in (2.5). Then we obtain

$$
\left\langle\varphi_{k}, \psi_{l}\right\rangle=a_{k}\left\langle\varphi_{k}, \psi_{l}\right\rangle+b_{l}\left\langle\varphi_{k}, \psi_{l}\right\rangle .
$$

Since $\left\langle\varphi_{k}, \psi_{l}\right\rangle$ does not vanish for any $k$ and $l$, we get from (2.6), $1=a_{k}$

(1) This proposition is due to Y. Saitō. 
$+b_{l}$. Putting $k=1$, we have $1=a_{1}+b_{l}$, and putting $l=1$, we have $1=a_{k}$ $+b_{1}$. This proves our proposition.

\section{BIBLIOGRAPHY}

[1] Coddington, E.A., and N. Levinson, Theory of ordinary differential equations, McGraw-Hill. New York (1955).

[2] Dunford, N., and J. Schwartz, Linear operators, part II, Interscience, New York (1963).

[3 ] Friedrichs, K.O., Criteria for discrete spectra, Comm. Pure Appl. Math. 3, (1950), 439-449.

[4] Gelfand, I.M., and B.M. Levitan, On the determination of a differential equation from its spectral function, Izv. Akad. Nauk SSSR, Ser. Math. 15, (1951), 309-360, (Russian). Amer. Math. Soc. Translations (2), 1, (1955), 253-304.

[ 5 ] Hilbert, D., Grundzüge einer allgemeinen Theorie der linearen Integralgleichungen, 4 Mittel., Nachr. d. Ges. d. Wiss. zu Göttingen. Math.-phys. Kl. 1906, (1906), 157-227.

[6] Ikebe, T., Orthogonality of the eigenfunctions for the exterior problem connected with -4 , Arch. Rational Mech. Anal. 19, (1965), 71-73.

[7] Krein, M.G., On the transfer function of a one-dimensional boundary problem of the second order, Dokl. Akad. Nauk SSSR (N. S.), 88, (1953), 405-408. (Russian)

[8] Levitan, B.M., On a uniqueness theorem. Dokl. Akad. Nauk SSSR (N. S.) 76, (1951), 485-488 (Russian)

[9] Marcenko, V.A., Concerning the theory of a differential operator of second order, Dokl. Akad. Nauk SSSR (N. S.) 72, (1950), 457-460. (Russian)

[10] Saitō, Y., Some remarks on orthogonality of generalized eigenfunctions for singular second-order differential equations, Publ. of Research Institute for Mathematical Sciences, Kyoto University, Ser. A, 2, (1966), 255-267.

[11] Sears, D.B., and E.C. Titchmarsh, Some eigenfunction formulae, Quart. J. Math. Oxford (2) 1, (1950), 165-175.

[12] Stone, M.H., Linear transformations in Hilbert space and their applications to analysis, Amer. Math. Soc. Colloquium Pub. 15, New York (1932).

[13] Titchmarsh, E.C., Eigenfunction expansions associated with second-order differential equations, part I, Second edition, Oxford University Press (1962).

[14] Weyl, H., Über gewöhnliche Differentialgleichungen mit Singularitäten und zugehörigen Entwicklungen willkürlichen Funktionen, Math. Ann. 68, (1910), 220-269.

[15] Yosida, K., On Titchmarsh-Kodaira's formula concerning Weyl-Stone's eigenfunction expansion, Nagoya Math. J. 1, (1950), 49-58., Correction, Nagoya Math. J. 6, (1953), 187-188. 